\title{
Plasma-catalysis: Is it just a question of scale?
}

\author{
J. Christopher Whitehead ( $₫)$ \\ School of Chemistry, The University of Manchester, Manchester M13 9PL, UK
}

(C) The Author(s) 2019. This article is published with open access at link.springer.com and journal.hep.com.cn 2019

\begin{abstract}
The issues of describing and understanding the changes in performance that result when a catalyst is placed into plasma are discussed. The different chemical and physical interactions that result and how their combination might produce beneficial results for the plasma-catalytic processing of different gas streams are outlined with particular emphasis being placed on the different range of spatial and temporal scales that must be considered both in experiment and modelling. The focus is on non-thermal plasma where the lack of thermal equilibrium creates a range of temperature scales that must be considered. This contributes in part to a wide range of inhomogeneity in different properties such as species concentrations and electric fields that must be determined experimentally by in situ methods and be incorporated into modelling. It is concluded that plasmacatalysis is best regarded as conventional catalysis perturbed by the presence of a discharge, which modifies its operating conditions, properties and outcomes often in a very localised way. The sometimes used description "plasma-activated catalysis" is an apt one.
\end{abstract}

Keywords plasma catalysis, plasma-activated catalysis, non-thermal plasma, $\mathrm{CO}_{2}$ conversion

\section{Introduction}

Plasma-catalysis, whereby in a material with catalytic properties in placed into or in close proximity with a gas discharge or plasma with the aim of achieving a beneficial improvement in the performance of the plasma processing of gas streams, has been a topic of research for almost a century. Kim et al. [1] has given an extensive history of the development of plasma-catalysis and pinpoints the first experiment where a catalyst was deliberately combined with an electrical discharge as being performed in 1921 by

Received September 4, 2018; accepted November 1, 2018

E-mail: j.c.whitehead@manchester.ac.uk
Ray and Anderegg [2]. They attempted to convert carbon monoxide to carbon dioxide by firstly using a silent discharge (as developed previously to produce ozone) in an atmospheric pressure mixture of oxygen and carbon monoxide and then passing the treated gases over a silver catalyst, observing that: "It is obvious that part of the oxidation takes place in the ozonizer while the gases are under the influence of the silent discharge and that part takes place while the gases are under the influence of the catalyst." The addition of a downstream Ag catalyst gave enhanced oxidation of the $\mathrm{CO}$ compared to experiments performed in 1879 by Berthelot [3] using a similar configuration but without a catalyst. A relatively small number of experiments were performed until the 1980's when there was a rapidly increasing number of reports motivated by the potential of plasma-catalysis to provide an efficient and sustainable process for a range of environmental, chemical and energy-related processes [4]. Recent review articles on plasma-catalysis will give a flavour of the range and nature of current research [1,5$16]$.

Initially, experiments yielded only averaged properties of the final states of the plasma-catalytic processing obtained by analytical methods applied to the processed gas streams or the catalyst when removed from reactor and the majority of current experiments still do. However, such experiments yield considerable and valuable information about the overall mechanism of the processing especially when the processing parameters of the plasma and the properties and composition of the gas and catalyst are systematically varied. The information about the underlying fundamental mechanism is generally obtained by intelligent and informed inference from the observed results sometimes supported by theoretical modelling or simulation based on some assumption about the physical and chemical behaviour of the plasma and catalyst involving gas dynamics, gaseous electronics, the chemical and physical properties of the catalyst and the supposed reaction mechanisms (gas and surface). Even at this stage, it quickly becomes clear that the mechanism of plasmacatalysis is a multivariable and complex interaction of 
many chemical and physical processes and properties that are hard to disentangle and understand. Using observables that are of bulk properties averaged over time and space is always going to limit the detail and hence the extent of understanding obtained.

We can obtain more information if we can look directly at the process whilst it is ongoing rather than looking at the final states of the processed gas and catalyst, which may have undergone some further change before their ex situ analysis thereby reducing the relevance of their analysis to gaining an understanding the mechanism of the process. Several groups are now able to explore the processing with in situ probing of the gases and the catalyst. This allows gaseous and surface-bound intermediates to be monitored whilst the processing is proceeding and gives fundamental information that is lacking from the "first generation" plasma-catalysis experiments. Indeed some of these experimental techniques can be configured in such a way as to provide spatial and temporal information as the processing takes place. This higher level of experimental information will enable us to probe the process at a more basic level and thereby gain a deeper understanding. This more detailed experimental information will provide confirmation of assumptions made by the modellers and allow their deductions about the mechanism of plasmacatalysis to be verified or not. In this way, our understanding will be obtained from an interpretation of the experimental data by inference or reasoning by analogy combined with a dynamical mechanism derived from established chemical and physical principles.

When considering the information that can be obtained, we need to consider the range of variables that can be studied in these processes. How do the properties of the gas flow, i.e., the gas dynamics and the chemical composition, the electrical properties of the discharge and the construction of the reactor affect the processing? What about the physical and chemical properties of the catalyst and its morphology? What is the range of distances and time scales that are appropriate to measure over? It is clear that different ranges of these variables will be appropriate to describe certain aspects of the mechanism. It is all a question of scale. For example, the fundamental chemical and physical dynamics of the processing can be considered at a molecular level with atomic distances, i.e., nanometre and nano- or sub-nanosecond time scales but also reaction times that can range from micro-second to several seconds as they depend on the gas flow rates and reaction cross sections. Physical processes including gaseous and surface diffusion, heat transfer and electrostatic properties such as gas breakdown, streamer propagation also require a range of distances that go from nanometre to the dimensions of the apparatus and time scales that go from sub-microsecond to minutes. The objective of this review is to examine the range of times and distances that we can or might expect to be able to measure over and where different processes relevant to plasma-catalysis lie on these scales.

\section{The interaction of plasma and catalyst}

To understand how plasma and catalyst might interact, we need to understand the nature of plasma and catalyst separately.

Essentially, plasma is an ionized gas containing an equal number of positive ions as of electrons and negative ions. It is thus electrically neutral and its degree of ionization can range from very low, i.e., partially ionized, to $100 \%$, or fully ionized. Technically, plasma can be created by a variety of discharge techniques involving chambers with electrodes energized by direct current, pulsed or alternating currents including electrodes screened by a dielectric material or using electrode-less systems such as inductively- or capacitively-coupled radio frequency radiation and microwaves. These discharges bring about a breakdown of the gas and create a range of species such as electrons, ions, dissociated and excited species giving plasma reactive properties that can potentially bring about chemical transformations. It can be characterized by the pressure regime in which it operates; either low, being less than atmospheric or high pressure, being atmospheric or higher. Low-pressure conditions favour surface collisions making catalytic effects easier to notice and they also minimise the deactivation of the excited states produced in the plasma through gas-phase.

Another distinction concerns the degree of thermal equilibrium in the plasma. It may be thermal with all the degrees of freedom including the electrons, ions and neutral species having the same temperature as the bulk gas (typically $>1000 \mathrm{~K}$ ). In contrast, non-thermal plasma has a high degree of disequilibrium between the light electrons and the heavier particles (ions, radical and molecules). Little kinetic energy is transferred between the light electrons and the heavy atomic and molecular species that remain close to their ambient temperature. Thus, a non-thermal discharge can create excited and reactive species that can normally only be produced in an equilibrium system such as an arc or flame at very high temperatures. Hence, plasma typically produces ionised, excited and reactive gaseous species in relatively low concentrations compared with the bulk neutral atomic and molecular species providing the means by which the key components in the gas stream are destroyed or converted into other potentially useful chemicals. In plasma-catalysis, these ionised, excited and reactive gaseous species can also interact with a catalyst at near ambient temperatures for which conventional thermal catalysis would be inactive. Typically, much of the research in plasmacatalysis has focussed on the use of atmospheric pressure, non-thermal plasma because of the simplification in 
engineering systems without the need for vacuum systems to obtain low pressures and the benefit that its low temperature operation minimises corrosion and deterioration of the catalyst through sintering or coking. In this review, I will mainly focus on the use of non-thermal plasma interacting directly with a catalyst.

Plasma-catalysis is a variant of heterogeneous catalysis where the gaseous phase of the reagents and products differs from the solid phase of the catalyst. Solid catalysts come in a variety of forms that all have the property of absorbing species onto their surface and thereby allowing possible migration into their bulk. Commonly, an active material such as a transition metal is placed onto a support material such as metal oxide or silica, a ceramic in a pressed or honeycomb form or zeolites. The role of the support is to provide a high surface area, with defined porosity and to give thermal and mechanical stability. The species that are adsorbed onto the catalyst can remain in their gaseous form upon adsorption and be weakly bound (called physisorption) or they may become dissociated into fragments and be strongly bound to the surface (chemisorption) and then react with either other species on the surface in a Langmuir-Hinshelwood process or by contact with an incoming gas-phase species via an Eley-Rideal mechanism. In contrast to thermal catalysis where the reactive species are formed dissociatively only on the surface of the heated catalyst, in plasma-catalysis reactive species can also be formed in the gas-phase either by dissociation within the plasma or by subsequent gas-phase reactions of plasma-excited species. The lifetime of these gaseous species that may become involved in an EleyRideal reaction with something adsorbed on the catalyst can range from a few nanoseconds in the case of electronically excited atoms or molecules through microseconds and seconds for radicals to several minutes for metastable species such as ozone. Electronically-excited species commonly encountered in plasma include atomic and molecular states of oxygen $\left(\mathrm{O}^{1} \mathrm{D}, E=1.967 \mathrm{eV}, \tau_{0}=\right.$ $\left.150 \mathrm{~s} ; \mathrm{O}_{2} a^{1} \Delta, E=0.977 \mathrm{eV}, \tau_{0}=64.6 \mathrm{~min}\right)$ and nitrogen $\left(\mathrm{N}^{2} \mathrm{D}, E=2.38 \mathrm{eV}, \tau_{0} \approx 17 \mathrm{~h} ; \mathrm{N}^{2} \mathrm{P}, E=3.576 \mathrm{eV}, \tau_{0} \approx 12 \mathrm{~s}\right.$; $\left.\mathrm{N}_{2} A^{3} \Sigma_{\mathrm{u}}, E=6.224 \mathrm{eV}, \tau_{0}=2.0 \mathrm{~s}\right)$. However, it is important to realize that at atmospheric pressure the mean free path between collisions is typically $100 \mathrm{~nm}$ and the time interval between collisions is $\sim 1 \mathrm{~ns}$. Thus the plasma-produced species may loose their energy by collisional quenching or be removed by gas-phase reactive collisions. The high reactivity of electronically excited atomic oxygen, $\mathrm{O}^{1} \mathrm{D}$, for example, reduces its lifetime from $150 \mathrm{~s}$ in the absence of collisions to $\sim 1 \mathrm{~ns}$ in a humid, atmospheric pressure, air plasma due to quenching by air molecules and reaction with water to form the hydroxyl radical, $\mathrm{OH}$.

The lifetime of the plasma-produced reactive species is a key parameter in determining their effectiveness in a plasma-catalysis reactor. For example, many plasmacatalytic processes involve oxidation where the key reagents are ground state oxygen atoms, $\mathrm{O}^{3} \mathrm{P}$, and hydroxyl radicals, $\mathrm{OH}$. The lifetime of a ground state oxygen atom, $\mathrm{O}^{3} \mathrm{P}$, in an atmospheric pressure plasma in dry air is $\sim 14 \mu \mathrm{s}$ [17] and that of $\mathrm{OH}$ depends on the gas composition but is typically $100 \mu \mathrm{s}$ in an air plasma but this reduces to $\sim 20 \mu \mathrm{s}$ in the presence of a potential reagent such as trichloroethylene [18]. This means that there will only be a narrow region or boundary layer immediately above the catalyst surface from which the short-lived plasma-created species can be adsorbed or participate in an Eley-Rideal type collision. Kim et al. [19] have calculated that for ground state oxygen atoms or $\mathrm{OH}$ radicals this layer will have a thickness of $\sim 50 \mu \mathrm{m}$. Outside of this layer, species produced by the plasma will react or be deactivated in the gas-phase in the same way that they do in the absence of the catalyst. This suggests that to gain the maximum effect from plasma-catalysis, the reactor must be designed and scaled in such a way that the plasma creates the reactive species very close to the surface of the catalyst (within a boundary layer of $\leqslant 50 \mu \mathrm{m}$ ). This could be achieved by using a porous material coated with the active catalyst through which the gas flows and the plasma is formed of surface discharges that propagate through the pores. Hansel [20] describes the formation of microdischarges inside the microporous ceramic foams. We shall discuss the criteria for propagation of plasma in porous material below. It is important that we can determine the spatial distribution of reactive species created in the volume of the plasma discharge especially those that exist close to the surface of the catalyst. Examples exist in the literature of the use of planar laser-induced fluorescence to determine such two-dimensional concentration profiles in plasma [21,22] and catalytic studies $[23,24]$ and it would be timely to make such measurements in a suitable plasma-catalysis system. Such spatially resolved information will generate valuable insight and provide important information upon which theoretical modeling can be based.

Recently, Christensen and co-workers [25] have suggested that there may also be a liquid-surface boundary layer involved in the process of plasma-catalysis where a liquid film could play an important role in the formation of species such as ketene and $\mathrm{C}_{2} \mathrm{O}_{5}$ when processing a $\mathrm{CO}_{2} /$ $\mathrm{CH}_{4} / \mathrm{N}_{2}$ mixture in a dielectric barrier discharge (DBD) with a Macor catalyst. The liquid film was found to be composed of many species including acetamide. As well as its effects on the chemistry, the liquid film also modifies the electric field experienced by the discharge as there is a change in the overall dielectric constant as the liquid film builds up. They make the intriguing suggestion that it might be possible to direct the plasma-induced chemistry that takes place at or near the plasma/liquid interface by adding a controlled amount of liquid thereby using the dielectric constant of the liquid to control the electric field at the interface.

In addition, it is also important that we know the identity of the species that are adsorbed onto the catalyst. Ideally, 
this also should be performed in situ in real time during the processing. In many plasma-catalysis studies, the catalyst is analysed by removing it from the reactor at various times during the processing and performing ex situ analysis of its surface using a range of surface analysis techniques such as infrared, Raman, thermal desorption spectroscopy and $\mathrm{X}$-ray photoelectron spectroscopy. But this only gives an indication of very strongly adsorbed species that remain on the surface long enough for remote analysis and will not identify the short-lived intermediates that may play an important role in the mechanism of plasma-catalysis. Recently, a range of techniques have been used for in situ detection of species at the surface in plasma-catalysis; one is diffuse reflectance infrared Fourier transform spectroscopy (DRIFTS) in which infrared radiation is reflected from the surface and collected allowing time-resolved analysis of the surface coverage of reagents, intermediates and products to be performed by identifying the vibrational frequencies characteristic of various chemical bonds and functional groups on the surface or immediately below the surface. An early application to plasma-catalysis can be found in the work of Stere et al. [26] who used DRIFTS to examine the selective catalytic reduction of NOx on a $\mathrm{Ag} / \mathrm{AlO}_{2}$ catalyst in the presence of toluene and $n$-octane where the catalyst was activated by an atmospheric pressure, helium plasma jet. Another techniques that also uses infrared spectroscopy but this time in transmission through a thin pellet of a catalyst, which is exposed to a DBD plasma has been applied to the plasma oxidation of acetone and isopropanol on a $\mathrm{CeO}_{2}$ catalyst [27]. Intermediates observed on the surface during the oxidation of acetone included isobutene and acetic acid whereas oxidation of isopropanol gave mainly adsorbed acetone, mesityl oxide and acetate. The dynamics of the plasmacatalytic processing in terms of the production and desorption of intermediates can be studied with a time resolution of $\sim 30 \mathrm{~s}$ which is determined by the Fourier transform infrared spectroscopy (FTIR) spectrometer.

Azzolina-Jury and Thibault-Starzyk [28] have used stepscan, time-resolved IR spectroscopy to study, in real-time, the formation of species both in the gas-phase and on the catalyst surface with a time resolution of $60 \mu \mathrm{s}$. Their work is a perfect example of the interplay involving plasmacreated species in the gas-phase and species adsorbed onto the catalyst and illustrates how both processes must be considered together. They have probed the mechanism of the plasma-assisted catalytic hydrogenation of $\mathrm{CO}_{2}$ on a nickel catalyst supported on a H-USY zeolite distinguishing between hydrogenation in the gas-phase and on the surface and identifying vibrationally-excited $\mathrm{CO}$ and $\mathrm{CO}_{2}$ formed in the plasma. A schematic example of this type of plasma-assisted catalytic mechanism for the hydrogenation of $\mathrm{CO}_{2}$ on a Ni-USY catalyst in a buffer gas of $\mathrm{Ar}$ at reduced pressure is shown in Fig. 1. CO was the major end product from the pulsed plasma dissociation of $\mathrm{CO}_{2}$ in the gas-phase. Desorbing $\mathrm{CH}_{4}$ and $\mathrm{H}_{2} \mathrm{O}$ were secondary products formed on the catalyst from successive hydrogenation of intermediate formates resulting from adsorption of vibrationally excited $\mathrm{CO}_{2}$ and of carbonyls from adsorption of the $\mathrm{CO}$ resulting from plasma-dissociation of $\mathrm{CO}_{2}$ in the gas-phase. Methane selectivity is reduced by the fact that $\mathrm{CO}$ is adsorbed onto the nickel covering the active sites and reducing the number of sites available for $\mathrm{CH}_{4}$ production. There was no evidence that plasma was

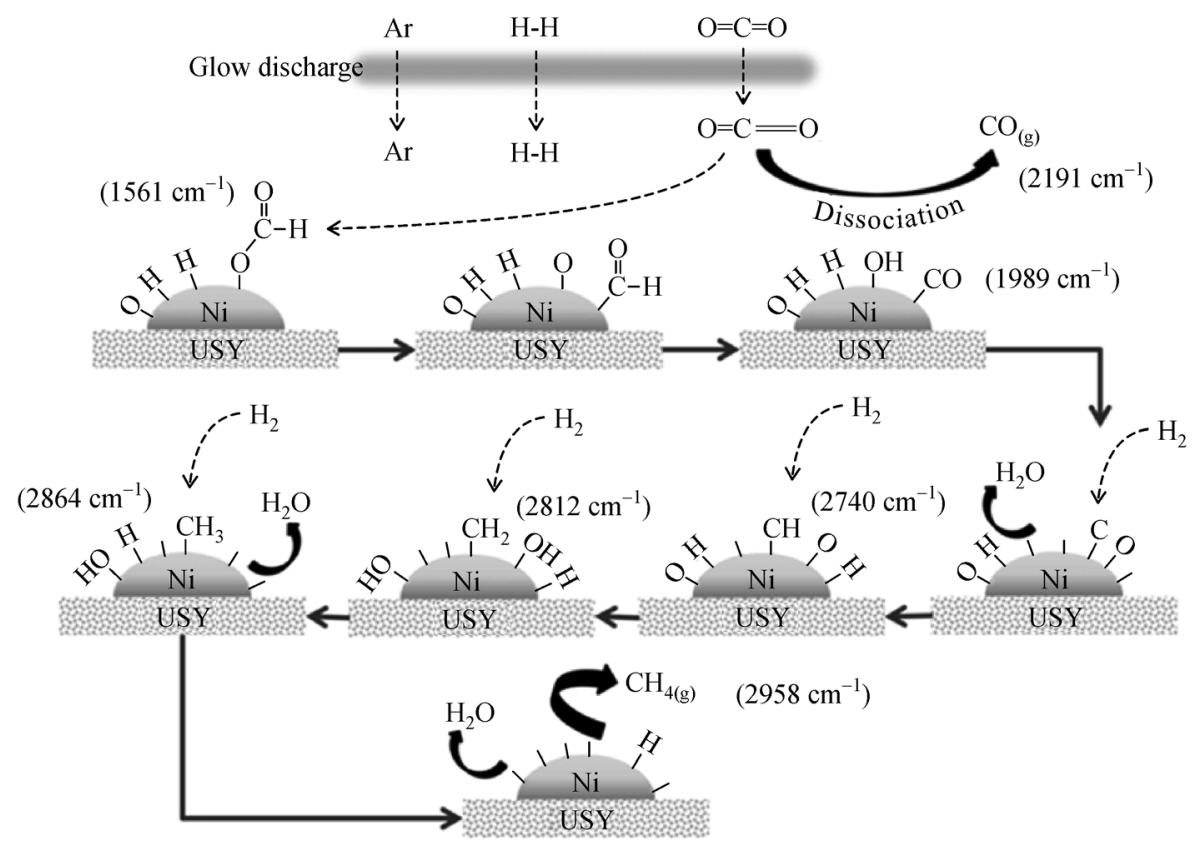

Fig. 1 A proposed mechanism for plasma-assisted $\mathrm{CO}_{2}$ hydrogenation over a Ni-USY zeolite. Reproduced from Ref. 28 with permission. Copyright 2017, Springer 
produced in the pores of the zeolite and it is thought that reaction is confined to the nickel.

\section{The catalytic landscape as seen by a plasma discharge}

At the end of the eighteenth and the beginning of the 19th century, the polymath, natural philosopher, botanist and explorer, Alexander von Humboldt, saw the connections between the natural and scientific worlds at a range of scales from the cosmic to the microscopic [29]. If he had had the power of electron microscopy and was observing the surface of a catalyst, he would have seen an immediate similarity with some of the landscapes that he had observed in his travels. Without the microscope, the surface of the catalyst might look flat and featureless but with the aid of the extra magnification afforded by the microscope, he would see that the surface was not smooth like a lake but had a terrain of peaks, slopes and steppes. It would contain islands of different sizes and there would be interesting openings that lead below the surface to tunnels and caves of differing sizes. On the catalyst, the islands would be the deposited particles of metal on a rough "sea" that was the substrate whose irregular features at the molecular and microscopic scale were of significance and the subterranean tunnels were the pores within the substrate. An illustration of the topography of the catalyst "landscape" can be seen in Fig. 2 reproduced from the paper by Neyts and Bogaerts [30]. It is this "landscape" that the plasma sees when it "looks" at the catalyst and the different features can cause distinct physical and chemical interactions that contribute to the overall process that we call plasma-catalysis. The plasma is essentially the atmosphere in which the landscape is placed.

One particular area of interest where we can see the effect of scale concerns the pores that lead from the surface into bulk of the catalyst. Pores in catalytic materials can selectively adsorb species depending on their size, which may affect the overall reactivity and selectivity of the plasma process. Penetration of plasma into the pores may specifically excite the adsorbed atoms or molecules creating localised reactive species. Kraus et al. [31] used $\mathrm{Ni}$ - and $\mathrm{Rh}$-coated, reticulated alumina ceramic foams inside a DBD reactor to study the dry reforming of methane with carbon dioxide. The foam was found to have a promoting effect on the chemistry that was attributed to the small pore size of $420 \mu \mathrm{m}$ increasing the breakdown field and giving an increased concentration of high-energy electrons producing enhanced destruction of the $\mathrm{CO}_{2}$. Experimental studies on the removal of pollutants from air [32] showed that for nanoporous materials (pore size $<0.8 \mu \mathrm{m}$ ), the electrical discharge does not penetrate into the pores but for mesopororus materials (pore size $\geqslant 15 \mu \mathrm{m})$ it is possible for microdischarges to penetrate into the pores and that excited species can be stabilised in the pores giving enhanced reaction times. Considerable insight on the mechanism and nature of plasma production within a pore has come from modelling studies. The criterion is that plasma can be produced in a pore if its diameter is greater than the Debye radius of the plasma [33]. The Debye radius is typically $>3 \mu \mathrm{m}$ in a microdischarge [34] although this value is dependent on the nature of the discharge gas and so we find that a microdischarge can be formed inside pores of $\mu \mathrm{m}$ scale as demonstrated by experiment. Modelling for He in a DBD,

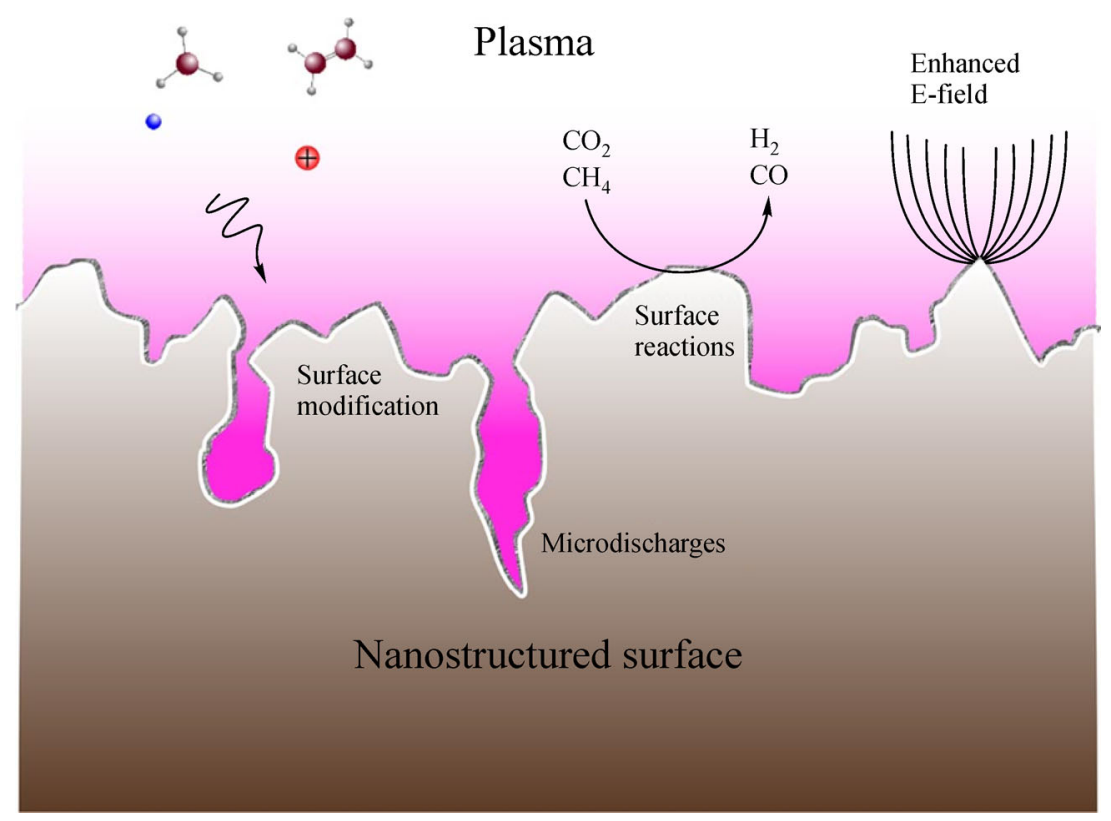

Fig. 2 A schematic representation of the cross-section of the gas-catalyst interaction region relevant to the plasma-catalytic conversion of the dry reforming of $\mathrm{CH}_{4}$ with $\mathrm{CO}_{2}$ into $\mathrm{CO} / \mathrm{H}_{2}$. Reproduced from Ref. 30 with permission. Copyright 2014, Institute of Physics 
at atmospheric pressure [25] shows that the electron density in and close to a $200 \mu \mathrm{m}$ pore is significantly enhanced and that for smaller pores (ca. $30 \mu \mathrm{m})$ an increase in charge density close to and in the pore can be achieved for high applied voltages. For nanoscale pores and an air plasma where the Debye radius is smaller for $\mathrm{He}$, it is found that there is only limited penetration for a pore diameter of $50 \mathrm{~nm}$ but that there is penetration within a 500 $\mathrm{nm}$ diameter pore for a high enough applied voltage [35]. The formation of reactive species by any plasma created within a pore will be limited by the ability of the precursors to diffuse into the pore. For an atmospheric pressure plasma Kim et al. [19] has shown that estimates of the rates of diffusion indicate that penetration into pores of nanometre dimension $(<2 \mathrm{~nm})$ is unfeasibly slow but that on a slightly larger scale $(2-50 \mathrm{~nm})$ it could be possible. Taken with the modelling results, it can be seen that plasma-generated species can be formed inside the pores of structured catalysts, which may interact with the catalyst surface and contribute to the plasma-catalyst interaction.

The "islands" of metal catalysts located on the substrate that the plasma can see, act as active sites upon which species formed in the plasma can be adsorbed and react or dissociate or else they can spill over onto the substrate and undergo further reaction before desorbing. Species can also be directly adsorbed onto the substrate and may produce different reaction products to those that are activated on the metal [36].

Surface irregularities on the catalyst or points of contact formed between the catalytic particles can cause enhanced electric fields when exposed to the plasma. These can give rise to local hotspots where a disproportionally high amount of the plasma-catalytic processing may take place [8]. Kim and co-workers [1] have discussed the timescale for heat transfer where the catalyst may be in the form of nanoscale metal particulates on a support material and this is summarized in diagrammatic form in Fig. 3. Because the metal particles $(10 \mathrm{~nm})$ are much smaller than the size of the support particles $(\sim 2 \mathrm{~mm})$, as energy is supplied to the catalyst by the plasma, the metal will be heated at a much more rapid rate than the support giving heating times of $10^{-12}$ and $5 \mathrm{~s}$, respectively. Thus, the metal particles will heat and cool much more rapidly than the support under pulsed plasma excitation. This indicates that in a nonthermal plasma multiple temperatures are needed to describe the species in the gas phase and on the catalyst that may vary with position within the reactor and also critically with time. In essence, there is a high degree of inhomogeneity with respect to time and position for many of the properties association with the plasma-catalytic process including the concentrations of the different species (including the electrons), the electron energies, the electric fields and the temperatures of surface features and of the different internal modes of the gaseous species.

The electrical properties of the catalytic material can also play an important role in how the plasma interacts with the catalyst. The use of dielectric materials can have a profound affect on the electrical properties of the discharge by changing the capacitance of the plasma reactor. This will influence the discharge breakdown voltage and the charge that can be transferred between the electrodes. In

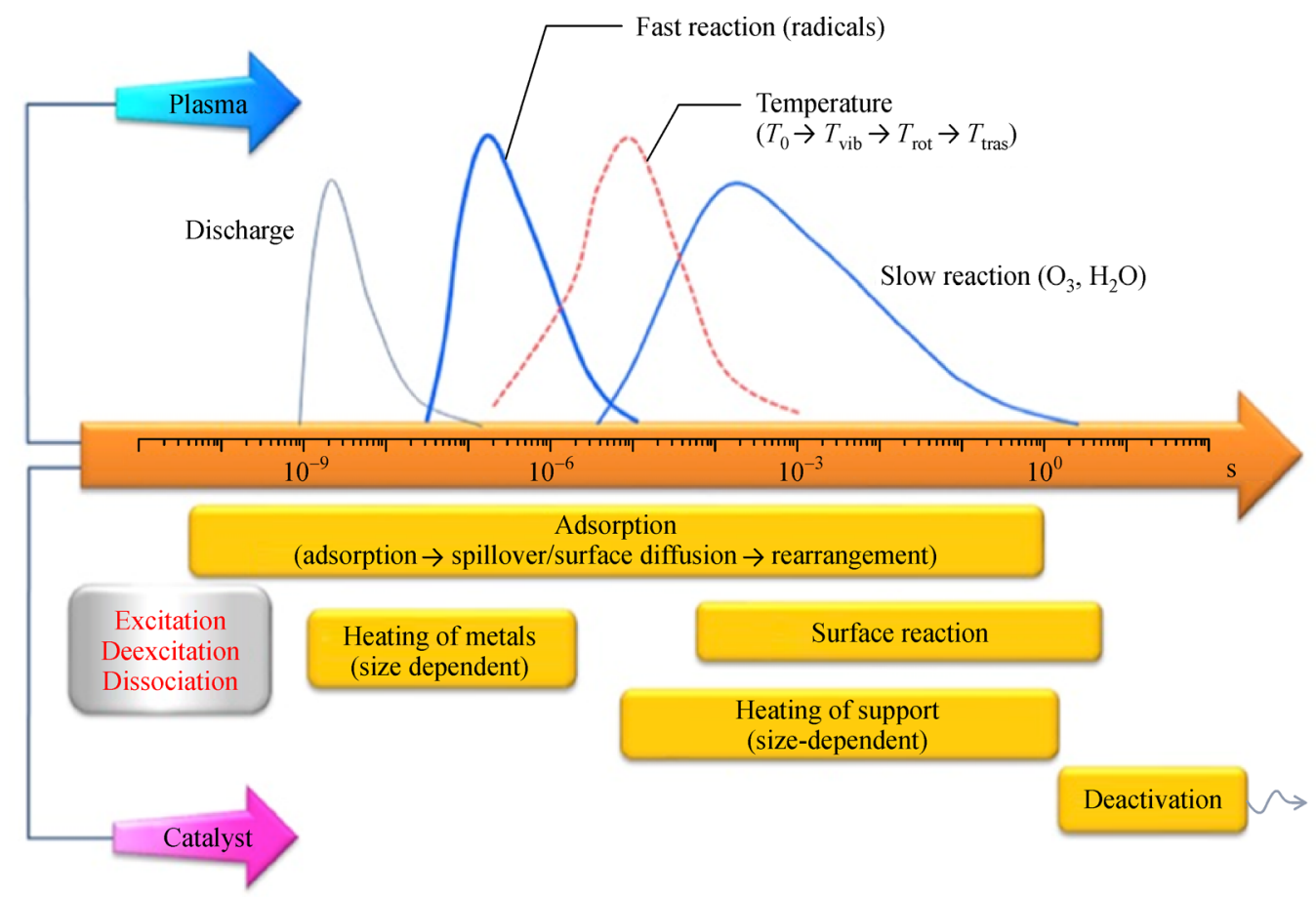

Fig. 3 Characteristic timescales in plasma-catalytic processes. Taken from Kim et al., Ref. 1, by permission. Copyright 2016, Springer 
general, more electrical energy can be deposited into a discharge when materials of higher dielectric constant are used resulting in the formation of more electrons of higher energy giving increased yield of ionised and excited species.

The nature of the packing of the plasma-catalytic reactor is important. For example, when a DBD reactor is filled with spherical beads of a high dielectric material such as $\mathrm{BaTiO}_{3}$ or $\mathrm{ZrO}_{2}$. Such materials are often ferroelectric and polarisation effects can cause enhanced electric fields at the points of contact between the beads giving higher concentrations of excited species. The nature of the discharge changes from streamers propagating in the gas when there is no packing to a mixture of gaseous and surface discharge when there is packing present. Increased conversion of $\mathrm{CO}_{2}$ is observed [37-40] in these arrangements and simulations [41,42] confirming that there are enhanced electric fields where the beads touch and the electron density is higher in these regions. This causes stronger and faster development of the plasma. The bead size is also important in affecting the conversion of the $\mathrm{CO}_{2}$ which increases as the bead size decreases as long as it is possible to sustain a discharge as the breakdown voltage for plasma action increases strongly as the size decreases [39]. Michielsen et al. [38] suggest that all these observations have wider implications for plasma-catalysis as the conversion obtained is not just a function of the active sites on the catalyst which is deposited on a support material but also on how that support material is packed into the reactor. There is a positive contribution from the packing to the conversion when using smaller sizes packing materials due to electric field enhancement at the contact points, but also a negative contribution resulting from the lower residence time of the gases because of the volume reduction at a given flow rate. Overall, this can give either a positive or negative effect depending on the nature of material inserted into the voids. This conclusion emphasises the important part played by the mechanical construction of a plasma-catalytic reactor and how it is packed. For example, it has been noted that using a DBD reactor that is only partially filled with a catalyst doubles the $\mathrm{CH}_{4}$ conversion and $\mathrm{H}_{2}$ yield during the dry reforming of $\mathrm{CH}_{4}$ with $\mathrm{CO}_{2}$ compared to a filled reactor [43].

The catalyst landscape is a hostile environment and is subjected to bombardment by ionised and energetic species including electrons and this will affect how the catalyst and its adsorbed species survive. It is well established that catalysts can be pre-treated by exposure to plasma for subsequent use in conventional thermal processing [4446]. For example, using a low pressure glow discharge or radio frequency plasma with $\mathrm{Ar}, \mathrm{He}, \mathrm{N}_{2}$ or air as a source of energetic electrons, it is possible to reduce a metal catalyst $[47,48]$. Plasma preparation can change the dispersion of metals on a catalyst, allowing for the treatment of sensitive low temperature materials and for the production of novel classes of catalysts involving selective doping and the preparation of nanoparticles. It is highly likely that if plasma can be used to prepare a catalyst in isolation under controlled conditions, then with the normal operating conditions of plasma-catalysis some of the plasma activity may result in a continual modification of the catalytic surface. Marinov et al. [49] have noted this for a $\mathrm{SiO}_{2}$ surface exposed to a low pressure nitrogen plasma commenting that the surface under contact with the plasma is not static and suggesting that this type of behaviour is expected for a range of surfaces and plasma chemistries. Recently, Gibson et al. [50] have studied the plasma-catalytic oxidation of methane using a $\mathrm{Pd} / \mathrm{Al}_{2} \mathrm{O}_{3}$ catalyst using X-ray absorption fine structure (XAFS) to give in situ structural information about catalyst during its exposure to plasma for the first time. They observed that there was no significant structural change to the catalyst but they were able to confirm from their XAFS data that there was heating of the metal in the range $150^{\circ} \mathrm{C}-200^{\circ} \mathrm{C}$ with the front of the catalyst bed being hotter than further downstream. This might be anticipated because the oxidation of methane is exothermic and its rate will be highest towards the start of the catalyst bed and similar temperature gradients in catalytic beds during plasmacatalytic processing due to reaction exo- or endothermicity have previously been observed using IR temperature measurements [51,52]. However, Gibson et al. [50] were able to show that the same degree of catalyst heating was observed in the absence of the methane leading them to conclude that only the plasma is responsible for the catalyst heating. Clearly the influence of the release of chemical and plasma energy as heat in plasma-catalysis needs further study but it is evident that there are important temperature gradients in a catalyst bed when activated by plasma that must be incorporated into our descriptions of the mechanism.

Finally, we should consider how the catalyst and its adsorbed species might behave when exposed to the electrons produced by the discharge. Electron-stimulated desorption is a well-known process in surface science. This can arise due to local heating of the surface by electron impact leading to thermal desorption. It is also possible that the addition of electrons can affect the bonding between a molecular adsorbate and the surface creating repulsive interactions leading to desorption. Bal et al. [53] have used density functional theory to look at the effect of negative surface charging of a catalyst exposed to a plasma. They examined the activation of $\mathrm{CO}_{2}$ on transition metal/alumina catalysts finding that the charging significantly enhances the dissociation of the $\mathrm{CO}_{2}$ to $\mathrm{CO}$ by improving the reductive power of the metal and that the magnitude of this effect differs for different metals. Dissociative electron attachment to a species on the surface giving an anion that then dissociates may also be an important process in the cased of electronegative species. It 
has been shown [54] that low energy electron attachment to a $\mathrm{CO}_{2}$ molecule adsorbed onto an oxygen vacancy on a $\mathrm{TiO}_{2}$ surface brings about dissociation of the resulting $\mathrm{CO}_{2}$ anion to give $\mathrm{CO}$ and the filling of the oxygen vacancy by the $\mathrm{O}^{-}$ion. The $\mathrm{CO}$ may then desorb or migrate to another region of the surface. Mei et al. [55] have developed this mechanism to describe the dissociation of $\mathrm{CO}_{2}$ on photocatlytic materials such as $\mathrm{BaTiO}_{3}$ and $\mathrm{TiO}_{2}$ where plasma-produced electrons of sufficient energy can create electron-hole pair states in an analogous method to the excitation of these materials by photons of the same energy in photocatalysis. It has also been shown that altering the amount of oxygen vacancies in perovskite materials by doping can be effective in increasing the efficiency of ethyl acetate oxidation using such materials as catalysts in a DBD reactor [56].

\section{Conclusions}

It is clear that determining the time-averaged bulk properties of the system can assess many of the gross attributes of plasma-activated catalysis such as yields of product, selectivities and energy efficiencies and how they vary with the operating conditions of the plasma and the choice and state of the catalyst. It is also clear that this level of information is often all that is needed to make decisions about how to optimise the system and engineer its scale-up. However, we need to be able to describe the structural and dynamic aspects of the plasma-catalyst aspects across a wide range of scales if we wish to be able to understand and explain the observations that we see in the bulk. Many of these effects have their origins in fundamental chemistry like the chemical composition of the catalyst and its properties such as electron structure, inter- and intramolecular interactions, its crystallinity and morphology. These are molecular properties and represent the lower limits of scale that we need to be able to study. We also need to understand and describe the properties of the bulk assembly of molecules that make up the catalyst and how this affect its electrostatic and electronic properties when subjected to the external and local fields associated with the plasma. This means that we need to cover distance scales that range from molecular $(\sim \mathrm{nm})$ through microscopic $(\sim \mu \mathrm{m})$ to the dimension of the reactor $(\sim \mathrm{cm})$. We must also consider the thermodynamics of the overall reaction being pursued by the plasma-catalyst combination and the heat transfer processes both in the gas and solid phases and the sources of heating and their associated timescales. The dynamics of the molecular processes such as electron-impact excitation, ionisation and dissociation due to the plasma in both the gas and surface phases must also be considered; these also include collisional processes such as reaction, energy transfer, adsorption and desorption and transport properties such as diffusion and migration.
This requires the full range of time scales from picoseconds to minutes as demonstrated by Kim et al. [1] in Fig. 3.

It is becoming apparent that understanding the mechanism of plasma-catalysis by regarding it as an additive effect of plasma acting alone and catalyst acting alone in the same system is too simplistic. It is far more complex than that: The addition or combination of the two techniques can bring about either a positive or negative overall effect; the former often being called synergistic. Plasma can bring about a reduced operating temperature for the catalytic process because non-thermal plasma can create at ambient temperatures high concentrations of species such as radicals, atoms and excited states that are normally only associated with high temperatures. The non-equilibrium nature and kinetics of the discharge sidesteps the normal demands of equilibrium thermodynamics. To begin to understand the mechanism of the interaction, I believe that we need to start with the catalyst and regard the plasma as a perturbation, discovering how the catalyst interacts at low temperature with the plasma-created species that are not normally present in conventional thermal catalysis but which may be key intermediates or represent novel reagents. In parallel, we must determine how the catalyst responds to the plasma in terms of its electrostatic properties affecting the spatial, temporal and energy distributions of ions and electrons and how the nature of the discharge might modify the electronic structure of the catalyst changing its binding properties with respect to adsorbed species. Many of the effects will be localised spatially and may also be temporally constrained. The effect of some of these interactions may be positive, some neutral and some negative compared with the normal catalytic process and the overall effect will then be the sum of the different interactions. The overall process is better described as "plasma-activated catalysis" or "plasmacatalyst coupling" rather than "plasma-assisted catalysis".

Neyts [15] has observed that plasma-catalysis is a complex process which is non-trivial to model as it poses the questions of "how to address the discrepancy in accessible time and length scales with respect to the experimental setup, and how can the different plasmaspecific factors be addressed?" Experimentalists can also ask exactly the same question as no single technique can give the full range of spatial and temporal resolution that we have identified as being necessary to describe plasma interactions with a solid catalyst in the presence of a gas stream. No single technique can probe all the properties that are required. In situ measurements are required to provide the fundamental information about the behaviour of the different species created in the gas-phase and on the catalytic surface that is necessary to test and develop the modelling. The modelling must also be able to incorporate the wide range of inhomogeneity in properties such as electric field, concentration, temperature etc. that are 
caused by the non-equilibrium nature of the plasma and the morphology of the catalyst. However, it is only by obtaining a fundamental understanding of these effects that we will be able to determine and specify the most effective composition and structure of catalysts for best performance under plasma activation and determine the optimal design, configuration and operating conditions for the plasma. The interaction between modellers and experimentalists is a vital part of understanding plasmacatalysis and thereby determining and developing the full potential of the technique.

Open Access This article is licensed under a Creative Commons Attribution 4.0 International License, which permits use, sharing, adaptation, distribution and reproduction in any medium or format, as long as you give appropriate credit to the original author(s) and the source, provide a link to the Creative Commons licence, and indicate if changes were made. The images or other third party material in this article are included in the article's Creative Commons licence, unless indicated otherwise in a credit line to the material. If material is not included in the article's Creative Commons licence and your intended use is not permitted by statutory regulation or exceeds the permitted use, you will need to obtain permission directly from the copyright holder. To view a copy of this licence, visit http://creativecommons.org/licenses/by/4.0/.

\section{References}

1. Kim H H, Teramoto Y, Ogata A, Takagi H, Nanba T. Plasma catalysis for environmental treatment and energy applications. Plasma Chemistry and Plasma Processing, 2016, 36(1): 45-72

2. Ray A B, Anderegg F O. The oxidation of carbon monoxide by passage with oxygen or air through the silent discharge and over ozone decomposing catalysts.1. Journal of the American Chemical Society, 1921, 43(5): 967-978

3. Berthelot P E M. Research on the ozone layer and the effuse electric. Compte Rendu, 1879, 88: 50-52 (in French)

4. Whitehead J C. Plasma-catalysis: Introduction and history. In: Tu X, Nozaki T, Whitehead J C, eds. Plasma Catalysis. Switzerland: Springer International Publishing AG, 2018, Chapter 1

5. Whitehead J C. Plasma catalysis for volatile organic compounds abatement. In: Handbook of Advanced Methods and Processes in Oxidation Catalysis. London: Imperial College Press, 2014, 155172

6. Whitehead J C. Plasma-catalysis: The known knowns, the known unknowns and the unknown unknowns. Journal of Physics. D, Applied Physics, 2016, 49(24): 243001

7. Snoeckx R, Bogaerts A. Plasma technology - a novel solution for $\mathrm{CO}_{2}$ conversion? Chemical Society Reviews, 2017, 46(19): 58055863

8. Neyts E C. Plasma-surface interactions in plasma catalysis. Plasma Chemistry and Plasma Processing, 2016, 36(1): 185-212

9. Neyts E C, Ostrikov K, Sunkara M K, Bogaerts A. Plasma catalysis: Synergistic effects at the nanoscale. Chemical Reviews, 2015, 115 (24): 13408-13446

10. Trinh Q H, Mok Y S. Environmental plasma-catalysis for the energy-efficient treatment of volatile organic compounds. Korean Journal of Chemical Engineering, 2016, 33(3): 735-748

11. Wang Z J, Jang B W L, Liu C J. Recent advances in plasma catalysis
(ISPCEM 2016). Topics in Catalysis, 2017, 60(12-14): 797-798

12. Chen G, Britun N, Godfroid T, Georgieva V, Snyders R, Delplancke-Ogletree M P. An overview of $\mathrm{CO}_{2}$ conversion in a microwave discharge: The role of plasma-catalysis. Journal of Physics. D, Applied Physics, 2017, 50(8): 084001

13. Feng X X, Liu H X, He C, Shen Z X, Wang T B. Synergistic effects and mechanism of a non-thermal plasma catalysis system in volatile organic compound removal: A review. Catalysis Science \& Technology, 2018, 8(4): 936-954

14. Wang B F, Xu X X, Xu W C, Wang N, Xiao H L, Sun Y H, Huang H $\mathrm{M}, \mathrm{Yu} \mathrm{L}, \mathrm{Fu} \mathrm{M} \mathrm{L}, \mathrm{Wu} J \mathrm{~L}$, et al. The mechanism of non-thermal plasma catalysis on volatile organic compounds removal. Catalysis Surveys from Asia, 2018, 22(2): 73-94

15. Neyts E C. Atomistic simulations of plasma catalytic processes. Frontiers of Chemical Science and Engineering, 2018, 12(1): 145154

16. Bogaerts A, Neyts E C. Plasma technology: An emerging technology for energy storage. ACS Energy Letters, 2018, 3(4): 1013-1027

17. Holzer F, Roland U, Kopinke F D. Combination of non-thermal plasma and heterogeneous catalysis for oxidation of volatile organic compounds Part 1. Accessibility of the intra-particle volume. Applied Catalysis B: Environmental, 2002, 38(3): 163-181

18. Hibert C, Gaurand I, Motret O, Pouvesle J M O H. (X) measurements by resonant absorption spectroscopy in a pulsed dielectric barrier discharge. Journal of Applied Physics, 1999, 85 (10): 7070-7075

19. Kim H H, Teramoto Y, Negishi N, Ogata A. A multidisciplinary approach to understand the interactions of nonthermal plasma and catalyst: A review. Catalysis Today, 2015, 256(1): 13-22

20. Hensel K. Microdischarges in ceramic foams and honeycombs. European Physical Journal D, 2009, 54(2): 141-148

21. Gao J L, Zhu J J, Ehn A, Alden M, Li Z S. In-situ non-intrusive diagnostics of toluene removal by a gliding arc discharge using planar laser-induced fluorescence. Plasma Chemistry and Plasma Processing, 2017, 37(2): 433-450

22. Vorac J, Hnilica J, Kudrle V, Dvorak P. Spatially resolved measurement of hydroxyl $(\mathrm{OH})$ radical concentration in a microwave plasma jet by planar laser-induced fluorescence. Open Chemistry, 2015, 13(1): 193-197

23. Saiki Y, Suzuki Y. Effect of wall surface reaction on a methane-air premixed flame in narrow channels with different wall materials. Proceedings of the Combustion Institute, 2013, 34(2): 3395-3402

24. Zhou J F, Blomberg S, Gustafson J, Lundgren E, Zetterberg J. Simultaneous imaging of gas phase over and surface reflectance of a $\operatorname{Pd}(100)$ single crystal during CO oxidation. Journal of Physical Chemistry C, 2017, 121(42): 23511-23519

25. Christensen P A, Ali A B, Mashhadani Z, Carroll M A, Martin P A. The production of ketene and $\mathrm{C}_{5} \mathrm{O}_{2}$ from $\mathrm{CO}_{2}, \mathrm{~N}_{2}$ and $\mathrm{CH}_{4}$ in a nonthermal plasma catalysed by earth-abundant elements: An in-situ FTIR study. Plasma Chemistry and Plasma Processing, 2018, 38(3): 461-484

26. Stere C E, Adress W, Burch R, Chansai S, Goguet A, Graham W G, Hardacre C. Probing a non-thermal plasma activated heterogeneously catalyzed reaction using in situ DRIFTS-MS. ACS Catalysis, 2015, 5(2): 956-964 
27. Jia Z, Rousseau A. Sorbent track: Quantitative monitoring of adsorbed VOCs under in-situ plasma exposure. Scientific Reports, 2016, 6(1): 31888

28. Azzolina-Jury F, Thibault-Starzyk F. Mechanism of low pressure plasma-assisted $\mathrm{CO}_{2}$ hydrogenation over Ni-USY by microsecond time-resolved FTIR spectroscopy. Topics in Catalysis, 2017, 60(1920): $1709-1721$

29. Wulf A. The Invention of Nature: The Adventures of Alexander von Humboldt, the Lost Hero of Science. London: John Murray, 2015

30. Neyts E C, Bogaerts A. Understanding plasma catalysis through modelling and simulation - a review. Journal of Physics. D, Applied Physics, 2014, 47(22): 224010

31. Kraus M, Eliasson B, Kogelschatz U, Wokaun A. $\mathrm{CO}_{2}$ reforming of methane by the combination of dielectric-barrier discharges and catalysis. Physical Chemistry Chemical Physics, 2001, 3(3): 294300

32. Roland U, Holzer F, Kopinke F D. Improved oxidation of air pollutants in a non-thermal plasma. Catalysis Today, 2002, 73(3-4): 315-323

33. Zhang Y R, Van Laer K, Neyts E C, Bogaerts A. Can plasma be formed in catalyst pores? A modeling investigation. Applied Catalysis B: Environmental, 2016, 185: 56-67

34. Fridman A. Plasma Chemistry. New York: Cambridge University Press, 2008, 142

35. Zhang Y R, Neyts E C, Bogaerts A. Enhancement of plasma generation in catalyst pores with different shapes. Plasma Sources Science \& Technology, 2018, 27(5): 055008

36. Mizushima T, Matsumoto K, Ohkita H, Kakuta N. Catalytic effects of metal-loaded membrane-like alumina tubes on ammonia synthesis in atmospheric pressure plasma by dielectric barrier discharge. Plasma Chemistry and Plasma Processing, 2007, 27(1): 1-11

37. Mei D, Zhu X, He Y L, Yan J D, Tu X. Plasma-assisted conversion of $\mathrm{CO}_{2}$ in a dielectric barrier discharge reactor: Understanding the effect of packing materials. Plasma Sources Science \& Technology, 2014, 24(1): 015011

38. Michielsen I, Uytdenhouwen Y, Pype J, Michielsen B, Mertens J, Reniers F, Meynen V, Bogaerts A. $\mathrm{CO}_{2}$ dissociation in a packed bed DBD reactor: First steps towards a better understanding of plasma catalysis. Chemical Engineering Journal, 2017, 326: 477-488

39. Butterworth T, Elder R, Allen R. Effects of particle size on $\mathrm{CO}_{2}$ reduction and discharge characteristics in a packed bed plasma reactor. Chemical Engineering Journal, 2016, 293: 55-67

40. Xu S, Whitehead J C, Martin P A. $\mathrm{CO}_{2}$ conversion in a non-thermal, barium titanate packed bed plasma reactor: The effect of dilution by Ar and $\mathrm{N}_{2}$. Chemical Engineering Journal, 2017, 327: 764-773

41. Zhang Y, Wang H Y, Jiang W, Bogaerts A. Two-dimensional particle-in cell/Monte Carlo simulations of a packed-bed dielectric barrier discharge in air at atmospheric pressure. New Journal of Physics, 2015, 17(8): 12

42. Koen Van L, Annemie B. Fluid modelling of a packed bed dielectric barrier discharge plasma reactor. Plasma Sources Science \& Technology, 2016, 25(1): 015002
43. Tu X, Whitehead J C. Plasma-catalytic dry reforming of methane in an atmospheric dielectric barrier discharge: Understanding the synergistic effect at low temperature. Applied Catalysis B-Environmental, 2012, 125: 439-448

44. Glonek K, Wroblewska A, Makuch E, Ulejczyk B, Krawczyk K, Wrobel R J, Koren Z C, Michalkiewicz B. Oxidation of limonene using activated carbon modified in dielectric barrier discharge plasma. Applied Surface Science, 2017, 420: 873-881

45. Liu L, Zheng C H, Wu S H, Gao X, Ni M J, Cen K F. Manganesecerium oxide catalysts prepared by non-thermal plasma for $\mathrm{NO}$ oxidation: Effect of $\mathrm{O}_{2}$ in discharge atmosphere. Applied Surface Science, 2017, 416: 78-85

46. Wang Z, Zhang Y, Neyts E C, Cao X X, Zhang X S, Jang B W L, Liu C J. Catalyst preparation with plasmas: How does it work? ACS Catalysis, 2018, 8(3): 2093-2110

47. Liu C J, Li M Y, Wang J Q, Zhou X T, Guo Q T, Yan J M, Li Y Z. Plasma methods for preparing green catalysts: Current status and perspective. Chinese Journal of Catalysis, 2016, 37(3): 340-348

48. Wang W, Wang Z, Wang J, Zhong C J, Liu C J. Highly active and stable Pt-Pd alloy catalysts synthesized by room-temperature electron reduction for oxygen reduction reaction. Advancement of Science, 2017, 4(4): 1600486

49. Marinov D, Guaitella O, de los Arcos T, von Keudell A, Rousseau A. Adsorption and reactivity of nitrogen atoms on silica surface under plasma exposure. Journal of Physics. D, Applied Physics, 2014, 47(47): 475204

50. Gibson E K, Stere C E, Curran-McAteer B, Jones W, Cibin G, Gianolio D, Goguet A, Wells P P, Catlow C R A, Collier P, Hinde P, Hardacre C. Probing the role of a non-thermal plasma (NTP) in the hybrid NTP catalytic oxidation of methane. Angewandte Chemie International Edition, 2017, 56(32): 9351-9355

51. Nozaki T, Okazaki K. Non-thermal plasma catalysis of methane: Principles, energy efficiency, and applications. Catalysis Today, 2013, 211: 29-38

52. Kameshima S, Tamura K, Mizukami R, Yamazaki T, Nozaki T. Parametric analysis of plasma-assisted pulsed dry methane reforming over Ni/ $\mathrm{Al}_{2} \mathrm{O}_{3}$ catalyst. Plasma Processes and Polymers, 2017, 14(6): 1600096

53. Bal K M, Huygh S, Bogaerts A, Neyts E C. Effect of plasmainduced surface charging on catalytic processes: Application to $\mathrm{CO}_{2}$ activation. Plasma Sources Science \& Technology, 2018, 27(2): 024001

54. Lee J, Sorescu D C, Deng X. Electron-induced dissociation of $\mathrm{CO}_{2}$ on $\mathrm{TiO}_{2}(110)$. Journal of the American Chemical Society, 2011, 133 (26): 10066-10069

55. Mei D, Zhu X, Wu C, Ashford B, Williams P T, Tu X. Plasmaphotocatalytic conversion of $\mathrm{CO}_{2}$ at low temperatures: Understanding the synergistic effect of plasma-catalysis. Applied Catalysis B: Environmental, 2016, 182: 525-532

56. Zhu X B, Zhang S, Yang Y, Zheng C H, Zhou J S, Gao X, Tu X. Enhanced performance for plasma-catalytic oxidation of ethyl acetate over $\mathrm{La}_{1-x} \mathrm{Ce}_{x} \mathrm{CoO}_{3+\delta}$ catalysts. Applied Catalysis B: Environmental, 2017, 213: 97-105 